\title{
Development of microelectrode arrays modified with inorganic-organic composite materials for dopamine electroanalysis
}

\author{
Stelian Lupu ${ }^{\mathrm{a}, *}$, Francisco Javier del Campo ${ }^{\mathrm{b}, *}$, Francesc Xavier Muñoz \\ a Department of Analytical Chemistry and Instrumental Analysis, Faculty of Applied Chemistry and Materials Science, University Politehnica of Bucharest, \\ Polizu Street 1-3, 011061 Bucharest, Romania \\ b Instituto de Microelectrónica de Barcelona, IMB-CNM (CSIC), Campus Universidad Autónoma de Barcelona, 08193 Bellaterra, Barcelona, Spain
}

\section{A R T I C L E I N F O}

\section{Article history:}

Received 25 August 2009

Received in revised form 27 November 2009

Accepted 3 December 2009

Available online $\mathrm{xxxx}$

\section{Keywords:}

Cyclic voltammetry

Electrochemical sensors

Microelectrode arrays

Dopamine

\begin{abstract}
A B S T R A C T
New electrode materials based on composite inorganic redox material-organic conducting polymer for electroanalysis of dopamine were developed. The composite inorganic-organic coatings based on Prussian blue (PB) and poly(3,4-ethylenedioxythiophene) (PEDOT) have been prepared by electrochemical methods onto gold disk microelectrode arrays. Both insoluble and soluble forms of PB were prepared in situ inside the organic matrix and their electrochemical behaviour was investigated in electrolyte solution. The composite inorganic-organic coating containing the soluble PB form exhibited good electrocatalytic activity towards dopamine (DA) oxidation in aqueous buffered solutions. The DA concentration was determined by cyclic voltammetry in the presence of a high excess of ascorbic acid (AA). A linear response for DA concentrations ranging from $10 \mu \mathrm{M}$ to $50 \mu \mathrm{M}$, in the presence of $1 \mathrm{mM}$ AA, with a sensitivity of $460 \mu \mathrm{A} / \mu \mathrm{M}$ and a detection limit of $4.3 \mu \mathrm{M}$, have been obtained.
\end{abstract}

(C) 2009 Published by Elsevier B.V.

\section{Introduction}

Electrochemical sensors and biosensors for the electroanalysis of biologically active compounds have attracted a great deal of interest due to their analytical performance. Electrochemical sensors provide low detection limits, a wide linear response range, and good stability and reproducibility, among other advantages. However, bare electrodes are more likely than chemically modified electrodes to suffer from interferences or surface fouling by products arising from follow-up reactions, associated to the main electrochemical process. The modification of electrode surfaces with inorganic or organic coatings often avoids these drawbacks and represents a rapid and versatile resource for the preparation of stable and selective new electrochemical sensors [1-5]. Inorganic coatings consisting of transition metal hexacyanoferrates, such as Prussian blue (PB, ferric ferrocyanide $\left.\mathrm{Fe}_{4}^{\mathrm{III}}\left[\mathrm{Fe}^{\mathrm{II}}(\mathrm{CN})_{6}\right]_{3}\right)$, have been used in the preparation of electrochemical sensors as well as amperometric biosensors [6-10]. PB is a mixed-valence compound that can be deposited onto the electrode surface as an electroactive film. There are two forms of $\mathrm{PB}$ that have been called "water insoluble PB", $\mathrm{Fe}_{4}^{\mathrm{III}}\left[\mathrm{Fe}^{\mathrm{II}}(\mathrm{CN})_{6}\right]_{3}$, and "water soluble PB", $\mathrm{KFe}^{\mathrm{III}}\left[\mathrm{Fe}^{\mathrm{II}}(\mathrm{CN})_{6}\right]$, respectively [11]. The soluble form of $\mathrm{PB}$ forms in the presence of

* Corresponding authors. Tel.: +40 21 4023886; fax: +40 213111796 (S. Lupu), tel.: +34 935947 700; fax: +34 935.801.496 (F.J. del Campo).

E-mail addresses: stelianl@yahoo.com (S. Lupu), franciscojavier.delcampo@ imb-cnm.csic.es (F.J. del Campo). an excess of potassium ions. The reduction of PB yields a colorless compound called Everitt's Salt (ES), while the oxidation of PB gives Berlin Green (BG). Pt/PB modified electrodes typically display two redox waves at ca. $0.2 \mathrm{~V}$ and $0.9 \mathrm{~V}$ vs. SCE, respectively. These waves correspond to the reduction of $\mathrm{PB}$ to ES and to the oxidation of $\mathrm{PB}$ to $\mathrm{BG}$, respectively. These redox processes can be described by using the two formula of PB, i.e. the soluble form (Eqs. (1) and (3)) and the insoluble form (Eqs. (2) and (4)), respectively, according to the following reactions $[11,12]$ :

- redox wave corresponding to the PB/ES system:

$\mathrm{KFe}^{\mathrm{III}}\left[\mathrm{Fe}^{\mathrm{II}}(\mathrm{CN})_{6}\right]+\mathrm{e}^{-}+\mathrm{K}^{+} \Longleftrightarrow \mathrm{K}_{2} \mathrm{Fe}^{\mathrm{II}}\left[\mathrm{Fe}^{\mathrm{II}}(\mathrm{CN})_{6}\right]$

$\mathrm{Fe}_{4}^{\mathrm{III}}\left[\mathrm{Fe}^{\mathrm{II}}(\mathrm{CN})_{6}\right]_{3}+4 \mathrm{e}^{-}+4 \mathrm{~K}^{+} \Longleftrightarrow \mathrm{K}_{4} \mathrm{Fe}_{4}^{\mathrm{II}}\left[\mathrm{Fe}^{\mathrm{II}}(\mathrm{CN})_{6}\right]_{3}$

- redox wave corresponding to the $\mathrm{PB} / \mathrm{BG}$ system:

$\mathrm{KFe}^{\mathrm{III}}\left[\mathrm{Fe}^{\mathrm{II}}(\mathrm{CN})_{6}\right]-\mathrm{e}^{-}-\mathrm{K}^{+} \Longleftrightarrow \mathrm{Fe}^{\mathrm{III}}\left[\mathrm{Fe}^{\mathrm{III}}(\mathrm{CN})_{6}\right]$

$\mathrm{Fe}_{4}^{\mathrm{III}}\left[\mathrm{Fe}^{\mathrm{II}}(\mathrm{CN})_{6}\right]_{3}-3 \mathrm{e}^{-}+3 \mathrm{Cl}^{-} \Longleftrightarrow \mathrm{Fe}_{4}^{\mathrm{III}}\left[\mathrm{Fe}^{\mathrm{III}}(\mathrm{CN})_{6} \mathrm{Cl}\right]_{3}$

Conducting polymers such as polypyrrole, polyaniline, polythiophene, and their derivatives, have been also used for the construction of electrochemical sensors based on modified electrodes. The preparation of composite inorganic-organic coatings capable of 
fast bulk and interfacial electron transfer during redox reactions is a method of choice for the construction of analytical devices. Various procedures for the preparation of jnorganic-organic composite material coatings have been recently reported [13]. Composite inorganic-organic coatings containing transition metal hexacyanoferrates and conducting polymers have been prepared in various configurations on electrode surfaces [14-17]. It has been demonstrated that the inorganic compound acts as a redox mediator, while the organic component plays the role of a stable matrix or a permselective membrane. By this approach, most stable, highly conducting, and selective coatings can be easily deposited over electrode surfaces. The choice of these components for the preparation of the composite materials is based on their unique properties, i.e. the excellent stability of the PEDOT layer in aqueous solution and its doped state [18-20], and the electrocatalytic activity of PB [21-23]. The electrochemical redox properties of each component are expected to produce a synergistic effect, taking into consideration that the PEDOT layer is in its conducting state in the potential region were the PB redox mediator displays good electrocatalytic activity.

This work reports the preparation of new electrode materials consisting of $\mathrm{PB}$ and poly(3,4-ethylenedioxythiophene) (PEDOT) deposited as thin films on gold disc microelectrode arrays is reported. We modified disk microelectrode arrays with two composite organic-inorganic materials, based on the soluble and the insoluble forms of PB in situ inside a PEDOT matrix. We then investigated the oxidation of DA at these modified microelectrode arrays by cyclic voltammetry (CV).

\section{Experimental}

\subsection{Chemicals}

All chemicals were used as received without any further purification. 3,4-ethylenedioxythiophene (EDOT, Aldrich) was used for the electrochemical preparation of the corresponding polymer. Distilled water was always used to prepare aqueous solutions.

\subsection{Electrochemical measurements}

The electrochemical experiments were carried out with an Autolab potentiostat/galvanostat $302 \mathrm{~N}$ (Ecochemie, The Netherlands) coupled to a PC running the GPES software, using a singlecompartment cell with three electrodes, at room temperature. The electrodes used were: a gold disk microelectrode array as working electrode, a saturated calomel electrode, SCE, as a reference electrode (Metrohm), and a platinum wire (Metrohm) as auxiliary electrode. Before each electrochemical measurement the surface of the working microelectrode array was electrochemically activated in $0.1 \mathrm{M} \mathrm{KCl}$ aqueous solution by holding the electrode potential at $0.0 \mathrm{~V}$ for $10 \mathrm{~s}$ followed by a second potential step at $-2.0 \mathrm{~V}$ for $10 \mathrm{~s}$. These potential steps were applied twice to activate the microelectrode arrays. The cyclic voltammograms were recorded simultaneously at both microelectrode arrays, using the BIPOT module of the potentiostat. The solutions were bubbled with high purity argon and a flow of argon was maintained over the solution during the measurements.

\subsection{Deposition procedure of $P E D O T-P B$ composite films}

The composite inorganic-organic coatings, i.e. PEDOT-PB-s and PEDOT-PB-i, respectively, have been prepared by a two-step method. For both coatings, the PEDOT layer was first electrogenerated from an aqueous solution containing $0.01 \mathrm{M}$ EDOT and $0.1 \mathrm{M}$ $\mathrm{K}_{3}\left[\mathrm{Fe}(\mathrm{CN})_{6}\right]$ as supporting electrolyte by potential cycling in the potential range from open circuit potential, $\mathrm{OCP}$, to $+1.0 \mathrm{~V}$ and reversed back to ${ }_{-} 0.6 \mathrm{~V}$, at a scan rate of $0.05 \mathrm{~V} \mathrm{~s}_{\hat{\Lambda}}^{-1}$ for five consecutive scans. The modified electrode obtained in the first step is referred to as Au/PEDOT-FeCN. In the second step, the Au/PEDOT-FeCN modified electrode was immersed in $2 \mathrm{M} \mathrm{FeCl}_{3}, 0.1 \mathrm{M}$ $\mathrm{KCl}$ and $0.01 \mathrm{M} \mathrm{HCl}$ aqueous solution and the electrode potential was scanned from 0.6 to $-0.2 \mathrm{~V}$ at a scan rate of $0.05 \mathrm{~V} \mathrm{~s}_{\wedge}^{-1}$ for five successive scans. The soluble PB is formed inside the PEDOT matrix during this potential cycling. The resulting modified electrode is denominated Au/PEDOT-PB-s. The composite coating containing the insoluble form of $\mathrm{PB}$ was prepared in a similar manner, except that $\mathrm{Na}_{4}\left[\mathrm{Fe}(\mathrm{CN})_{6}\right]$ was used as supporting electrolyte in the first deposition step, and no $\mathrm{KCl}$ was used in the second step. The absence of the potassium ions in these deposition solutions results in the formation of the insoluble form of PB. The resulting modified electrode is referred as to Au/PEDOT-PB-i.

\subsection{Fabrication of microelectrode arrays}

The microelectrode arrays used in this work comprise 816 microelectrodes of $10 \mu \mathrm{M}$ radius. They are arranged in a $24 \times 34$ square lattice and present a centre-to-centre separation of $50 \mu \mathrm{M}$ along both the $x$ and $y$ axes. These microelectrode arrays were fabricated on silicon chips using standard microfabrication techniques, as described in previous works [24], but a short summary will be given here for convenience. After growing a $1 \mu \mathrm{M}$ thick layer of thermal oxide over a silicon wafer, a triple metal layer consisting of $\mathrm{Ti}(25 \mathrm{~nm}), \mathrm{Ni}(25 \mathrm{~nm})$ and $\mathrm{Au}(100 \mathrm{~nm})$ is deposited. The gold areas where the microelectrodes and the contacts will be defined are etched in a wet step following a photolithographic step. This photolithographic step was performed insolating a positive resist (Clariant AZ6512) through a suitable dark field mask. After the metal has been etched, the excess resin is stripped in acetone and the wafers are thoroughly rinsed. Next a passivation layer is deposited over the wafers to protect the connection pads and also to help define the final geometry of the devices. This passivation layer is a mixed layer of silicon oxide $(700 \mathrm{~nm})$ and silicon nitride $(400 \mathrm{~nm})$. The contact pads and the microelectrodes are open through this passivation layer by reactive ion etching after a second photolithographic step. Once the contacts are open and the microelectrodes have been defined, the excess resin is stripped and the wafers rinsed. The wafers are then diced into individual chips and mounted on suitable printed circuit boards, where they are wire bonded and encapsulated.

\section{Results and discussion}

3.1. Preparation and characterization of microelectrode array modified

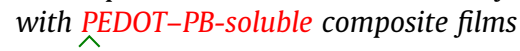

Microelectrode arrays were activated according to the procedure described in the Experimental section and then were characterized in aqueous solution containing a redox probe. Fig. 1 shows typical cyclic voltammograms recorded at two microelectrode arrays on a chip in $1 \mathrm{mM} \mathrm{K} \mathrm{K}_{3} \mathrm{Fe}(\mathrm{CN})_{6}$ and $0.1 \mathrm{M} \mathrm{KCl}$ aqueous solution at a potential scan rate of $0.05 \mathrm{~V} \mathrm{~s}^{-1}$. From the voltammograms, a population of active microdiscs around $50 \%$ was determined. This low yield was due to an imperfect etching of the silicon oxide layer used to pattern the electrodes during their fabrication.

Peak-shaped voltammograms were obtained due to the heavy overlap of the diffusion layers of neighbouring electrodes within the array at the potential scan rate used for voltammetric measurements. The voltammograms are very reproducible, attesting that the arrays behave similarly under these experimental conditions. Once the array was activated and its proper functioning checked, 


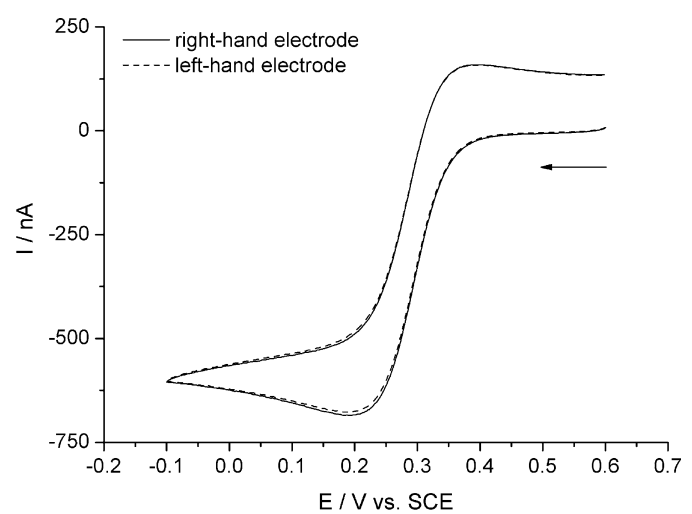

Fig. 1. Cyclic voltammograms recorded at the two bare Au microdisk electrode arrays in one chip (dotted and solid line) in $1 \mathrm{mM} \mathrm{K}_{3} \mathrm{Fe}(\mathrm{CN})_{6}$ and $0.1 \mathrm{M} \mathrm{KCl}$ aqueous solution at a potential scan rate of $0.05 \mathrm{~V} \mathrm{~s}^{-1}$.

one of the arrays was covered with a pure PEDOT organic coating (see Fig. 2A) while the other array was modified with the PEDOT-FeCN composite coating (see Fig. 2B). Fig. 2A shows that the polymerisation of PEDOT occurs in the potential range from 0.75 to $1.0 \mathrm{~V}$ vs. SCE, and that the PEDOT layer grows on the electrode surface. The shape of the cyclic voltammograms is similar to that of those recorded on conventional size electrodes and microelectrodes $[25,26]$.

The presence of the inorganic redox mediator results in a peakshaped voltammogram (see Fig. 2B). The electrochemical polymerisation occurs in the presence of ferricyanide ions and thus the resulting organic polymer is doped with counterions from the electrolyte solution.

After the deposition of PEDOT-FeCN coating, the modified electrode array was immersed in $\mathrm{Fe}^{3+}$ containing solution in order to prepare the "soluble" PB layer. Fig. $2 \mathrm{C}$ reports the cyclic voltammograms recorded at Au/PEDOT-FeCN modified electrode in aqueous solution containing $0.002 \mathrm{M} \mathrm{FeCl}_{3}, 0.1 \mathrm{M} \mathrm{KCl}$ and $0.01 \mathrm{M} \mathrm{HCl}$.

Note the presence of a redox wave characterized by an anodic peak at ca. $+0.3 \mathrm{~V}$ and a cathodic peak at ca. $+0.27 \mathrm{~V}$, respectively. This redox wave is ascribed to the PB/ES redox system and it is very stable as it can be seen from cyclic voltammograms recorded for five potential scans. Thus the in situ formation of the soluble form of PB is clearly demonstrated. This modified electrode was then immersed in phosphate buffer solution (PBS) and characterized by cyclic voltammetry (see Fig. 3A). The composite coating is very stable in neutral buffered aqueous solution. This is in contrast to PB-only films, which rapidly decompose in such media. Fig. 3B shows the cyclic voltammogram recorded at the "reference" $\mathrm{Au} /$ PEDOT modified microelectrode array from the chip. From Fig. 3B it can be observed a redox wave at ca. $+0.15 \mathrm{~V}$ that is ascribed to the polaron formation and the corresponding doping-dedoping redox processes. The stability of this wave is similar to those recorded on microelectrode disks and it is more visible than in the case of conventional size electrodes in such buffered solutions. The modified array was further characterized in array mode in a potassium phosphate buffer solution (PBS) and the corresponding voltammograms are depicted in Fig. $3 C$. It is important to note the successful modification of each electrode in the array as well as their electrochemical behaviour in the absence of the analyte. As to the polaron peak in the PEDOT coating, these measurements
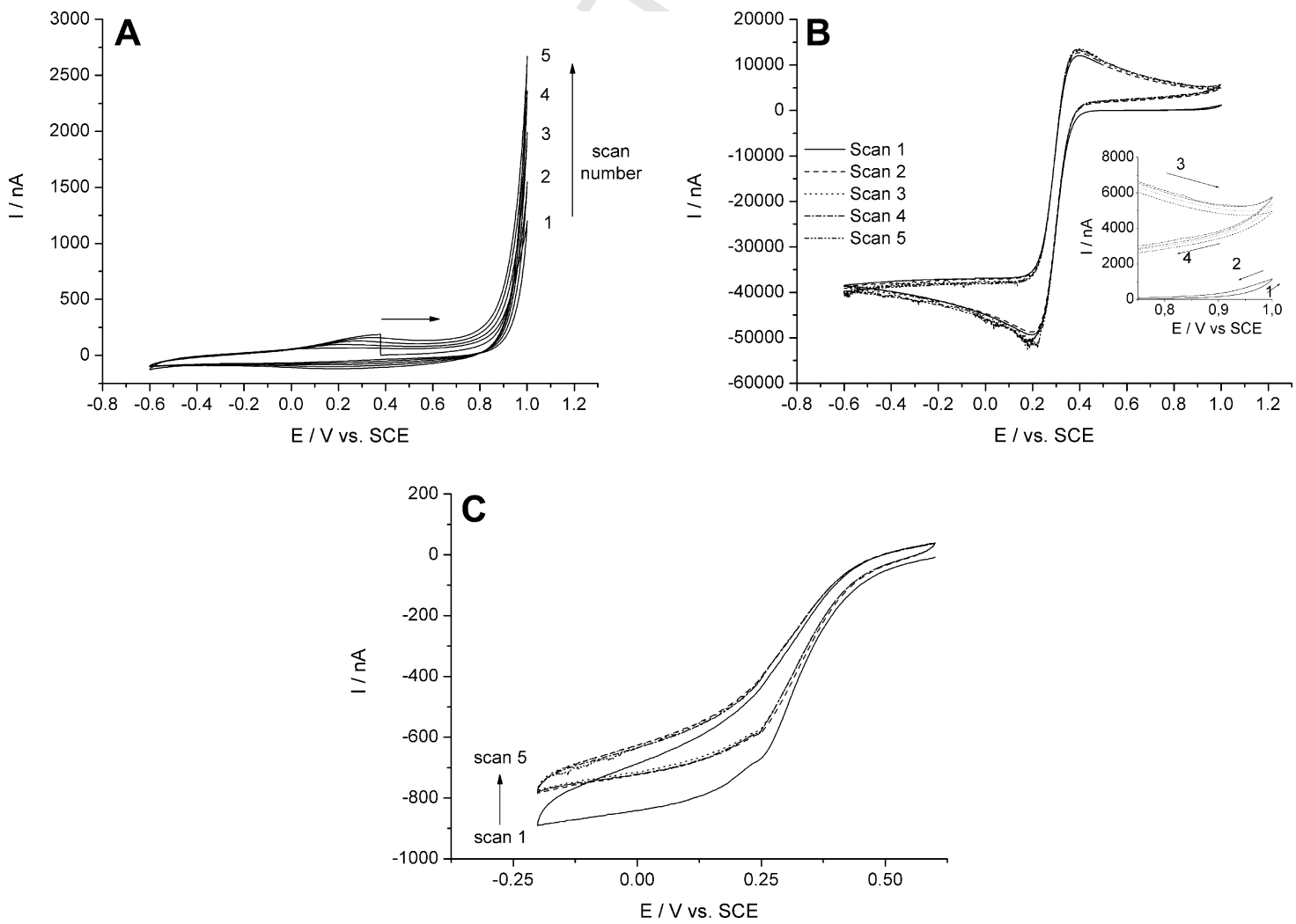

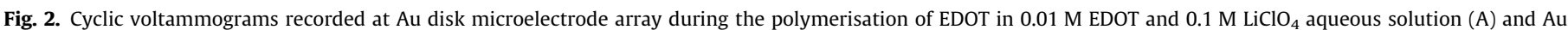

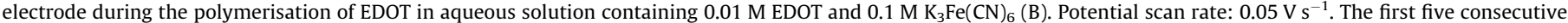

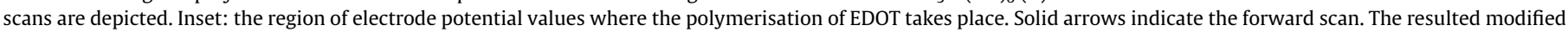

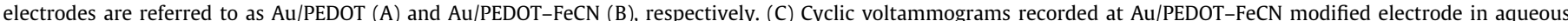

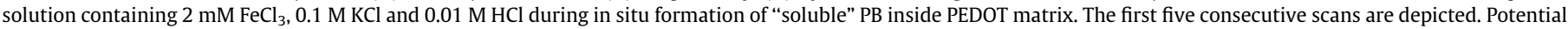
scan rate: $0.05 \mathrm{~V} \mathrm{~s}^{-1}$. The resulting modified electrode is denoted as Au/PEDOT-PB-s. 

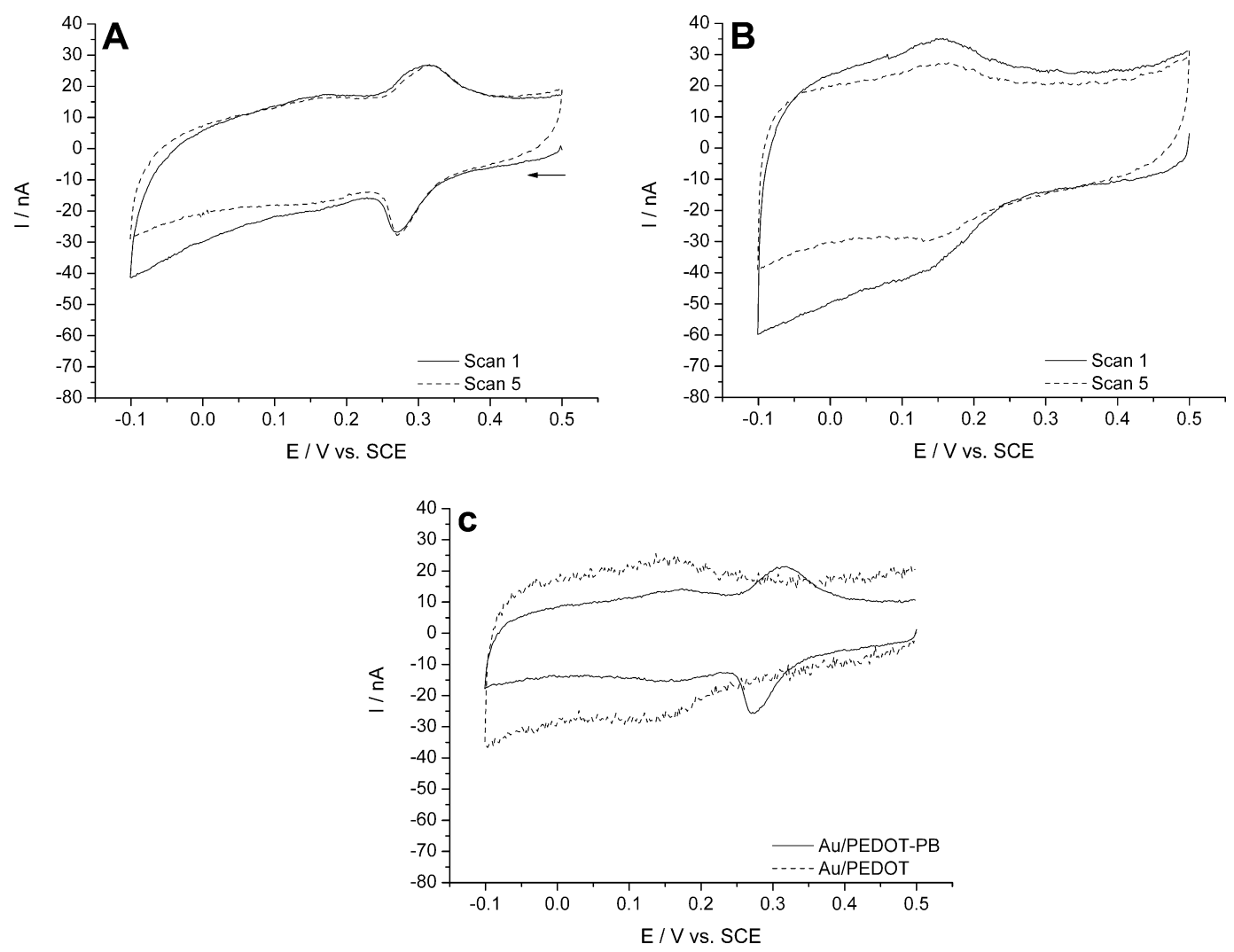

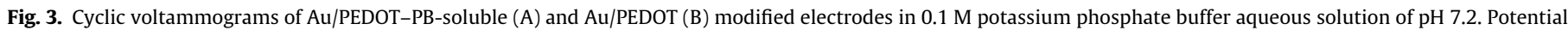

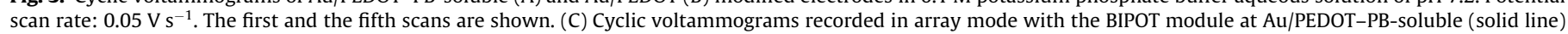

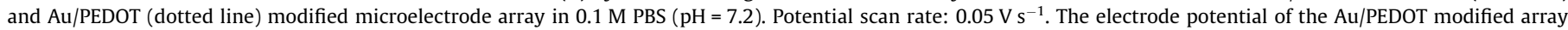
follows that of the array modified with the Au/PEDOT-PB-soluble layer.

clearly indicate a slight shift of the polaron wave towards more positive values in the case of the PEDOT-PB composite coating. Moreover, the cyclic voltammograms show that the presence of the inorganic redox material increases the conductivity of the coating, i.e. there is a less capacitive like shape for the PEDOT-PB corresponding cyclic voltammograms. The electrochemical redox properties of the composite material are mainly dominated by the inorganic redox mediator. In the investigated potential range, the PEDOT coating is in its conducting state and it is porous enough to permit the flux of potassium ions, necessary to maintain the electroneutrality and to afford the proper redox functioning of the PB component. The positively charged PEDOT matrix facilitates the incorporation of the negatively charged inorganic component $\mathrm{PB}$ and assures the excellent stability of the composite material in aqueous buffered solution. These electrochemical properties of the composite materials result in cyclic voltammograms that clearly show the contribution of each component to the overall behaviour of the coating.

\subsection{Preparation and characterization of microelectrode array modified} with PEDOT-PB-insoluble composite films

The preparation and the electrochemical characterization of PEDOT-PB-insoluble composite coating deposited as thin film onto Au disk microelectrode arrays were also studied. Fig. 4A shows cyclic voltammograms recorded at one of the Au electrodes in the chip during the electrochemical polymerisation of EDOT in the presence of sodium ferrocyanide. This redox mediator was chosen to avoid the presence of potassium ions during the preparation of the composite coating.
There is a small increase of current in the potential range between 0.75 and $1.0 \mathrm{~V}$ vs. SCE due to the electrochemical polymerisation of EDOT. The presence of the inorganic redox mediator results in a peak-shaped voltammogram. During the electrochemical polymerisation of EDOT, the ferrocyanide ions are incorporated into the organic matrix. The resulting modified electrode is referred as to Au/PEDOT-FeCN-Na. This is the basis for the preparation of insoluble PB form. The second microelectrode array was used as substrate for the electrochemical deposition of pure PEDOT layer, and was considered as "reference" coating with respect to the composite one. The shape of the CVs is similar to those presented in Fig. 2A. Also, the current increases in the potential range 0.75-1.0 V due to the deposition of the PEDOT layer. This modified array is referred to as Au/PEDOT. After the deposition of the PEDOT-FeCN-Na coating, the Au/PEDOT-FeCN-Na electrode was immersed in $\mathrm{Fe}^{3+}$ containing aqueous solution so that the insoluble form of PB may form inside the PEDOT matrix (see Fig. 4B). In this case there is no redox wave associated with the $\mathrm{PB} / \mathrm{ES}$ redox system due to the absence of the potassium ions in both the deposition solution and the transfer solution. It is well known that the $\mathrm{PB} / \mathrm{ES}$ redox wave is evidenced only in potassium containing aqueous solution [12]. This modified electrode is referred as to Au/PEDOT-PB-insoluble. This Au/PEDOT-PB-insoluble modified electrode was then characterized in sodium phosphate buffer aqueous solution. The voltammograms in Fig. 4C (solid line) lack the redox wave characteristic to the $\mathrm{PB} / \mathrm{ES}$ redox system because potassium ions were absent from the preparation and characterization solutions. As to the "reference" modified electrode, Fig. 4C (dotted line) shows that the electrochemical polymerisation of EDOT was successful, because the redox wave corresponding to the polaron formation is visible. Next, the Au/PEDOT-PB-insoluble modified 

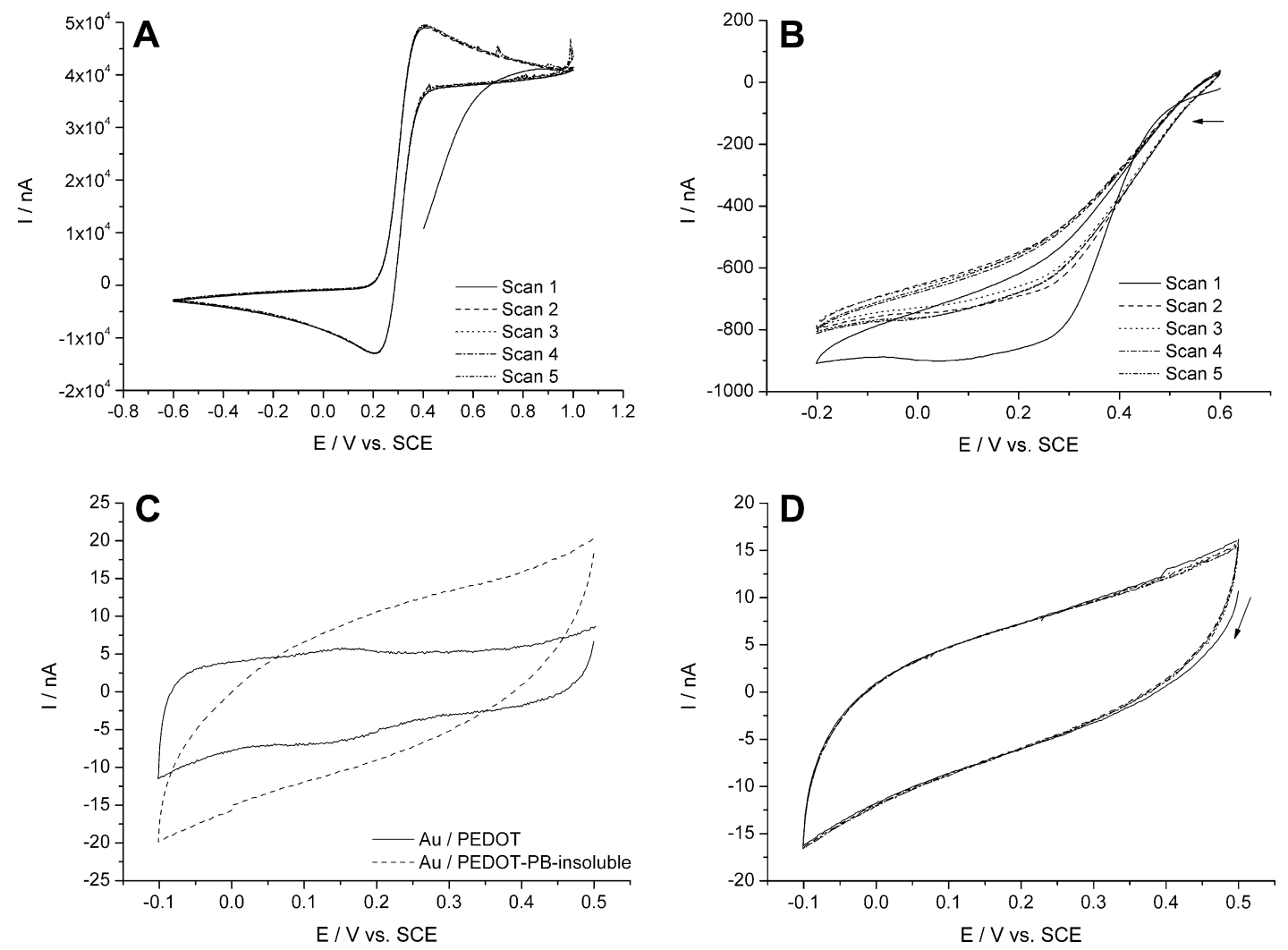

Fig. 4. (A) Cyclic voltammograms recorded at Au microelectrode array in aqueous solution containing $0.01 \mathrm{M} \mathrm{EDOT}$ and $0.1 \mathrm{M} \mathrm{Na}_{4} \mathrm{Fe}(\mathrm{CN})_{6}$. Potential scan rate: $0.05 \mathrm{~V} \mathrm{~s}{ }^{-1}$. The first five consecutive scans are depicted. (B) Cyclic voltammograms recorded at Au/PEDOT-FeCN-Na array in aqueous solution containing $2 \mathrm{mM} \mathrm{FeCl}_{3}$, and $0.01 \mathrm{M} \mathrm{HCl}_{\text {. }}$ Potential scan rate: $0.05 \mathrm{~V} \mathrm{~s}^{-1}$. The first five scans are represented. (C) Cyclic voltammograms recorded at Au/PEDOT-PB-insoluble (dotted line) and Au/PEDOT (solid line) modified electrodes in $0.1 \mathrm{M}$ sodium phosphate buffer solution. (D) The first five successive scans recorded at Au/PEDOT-PB-insoluble modified electrode in potassium phosphate buffer solution of $\mathrm{pH}=7.2$. Potential scan rate: $0.05 \mathrm{~V} \mathrm{~s}^{-1}$.

electrode was immersed in $0.1 \mathrm{M}$ potassium phosphate buffer aqueous solution and investigated by CV (see Fig. 4D). Note that presence of potassium ions in high concentration does not allow the conversion of the PB insoluble form into the soluble one. However, this conversion is observed in the case of $\mathrm{PB}$ only modifying layer and it is not fully reversible once the PB layer has been in contact with electrolyte solution containing other cations, like $\mathrm{Na}^{+}, \mathrm{Li}^{+}$, $\mathrm{Cs}^{+}[12,27,28]$. In our case, a high concentration of sodium ions during the preparation of the composite material may block the channels in the zeolitic structure of $\mathrm{PB}$, complicating their later replacement by potassium ions.

\subsection{Analytical applications of PEDOT-PB-soluble modified array in the voltammetric determination of dopamine (DA)}

The modified array was used in the voltammetric determination of DA in neutral buffered aqueous solution. Fig. 5A reports the CVs recorded simultaneously at both microelectrode arrays on a chip.

The PEDOT-PB-soluble modified array showed excellent analytical performance in the voltammetric determination of DA. The sensors responds to low DA concentrations, i.e. from 2 to $10 \mu \mathrm{M}$, by using cyclic voltammetry as detection method, and these low DA concentrations can be usually achieved only by using differential pulse and square wave voltammetry $[10,20]$. The sensor showed a linear response according to the equation: $i_{\mathrm{pa}}(\mathrm{nA})=$ $1.2 C_{\mathrm{DA}}(\mu \mathrm{M})+3.6$, with a correlation coefficient of $R^{2}=0.9966$ (see inset of Fig. 5A), and a detection limit of $2 \mu \mathrm{M}$. Surprisingly, the PEDOT-only coating showed no electrocatalytic activity towards DA oxidation. Therefore these results clearly demonstrate the benefit of using the PEDOT-PB-soluble modified microelec- trode array. The voltammetric determination of DA was also performed in the presence of a strong interferent like ascorbic acid (AA). It is well known that AA strongly influences the voltammetric determination of DA, and therefore we choose to study the electrocatalytic efficiency of the composite coating for simultaneous determination of DA in the presence of AA. Fig. 5B reports the cyclic voltammograms of the PEDOT-PB-soluble modified array recorded during the voltammetric determination of DA in the presence of an excess of $1 \mathrm{mM}$ AA. The DA oxidation produces an anodic wave at $0.28 \mathrm{~V}$, while the AA oxidation occurs at $0.1 \mathrm{~V}$ that is a peak potential separation of ca. $180 \mathrm{mV}$ was obtained, as it can be seen from the square wave voltammogram depicted in the inset of Fig. 5B. The large peak potential separation obtained at the modified microelectrode array is attributed to the electrostatic interaction between the analytes and the composite coating. At physiological $\mathrm{pH}$, DA exists as a cation and AA as an anion. The potential shift towards negative potential values observed for AA oxidation can be ascribed to the electrostatic interaction of the ascorbate anions with the polarons. It is well known that conducting polymers can act as redox mediators and the electrocatalytic activity arises from the formation of polarons, that is of positive charges on the polymer backbone. Therefore, the oxidation of AA is facilitated at PEDOT-modified electrodes by the negative charge of the molecule, while oxidation of positively charged species, like DA, could be made more difficult. On the other hand, the presence of the negatively charged inorganic redox mediator inside the PEDOT matrix could influences the oxidation of DA, by reducing the oxidation potential in comparison with an unmodified electrode. Therefore, the electrochemical response of the modified array towards both analytes can be considered as a result of a synergistic 

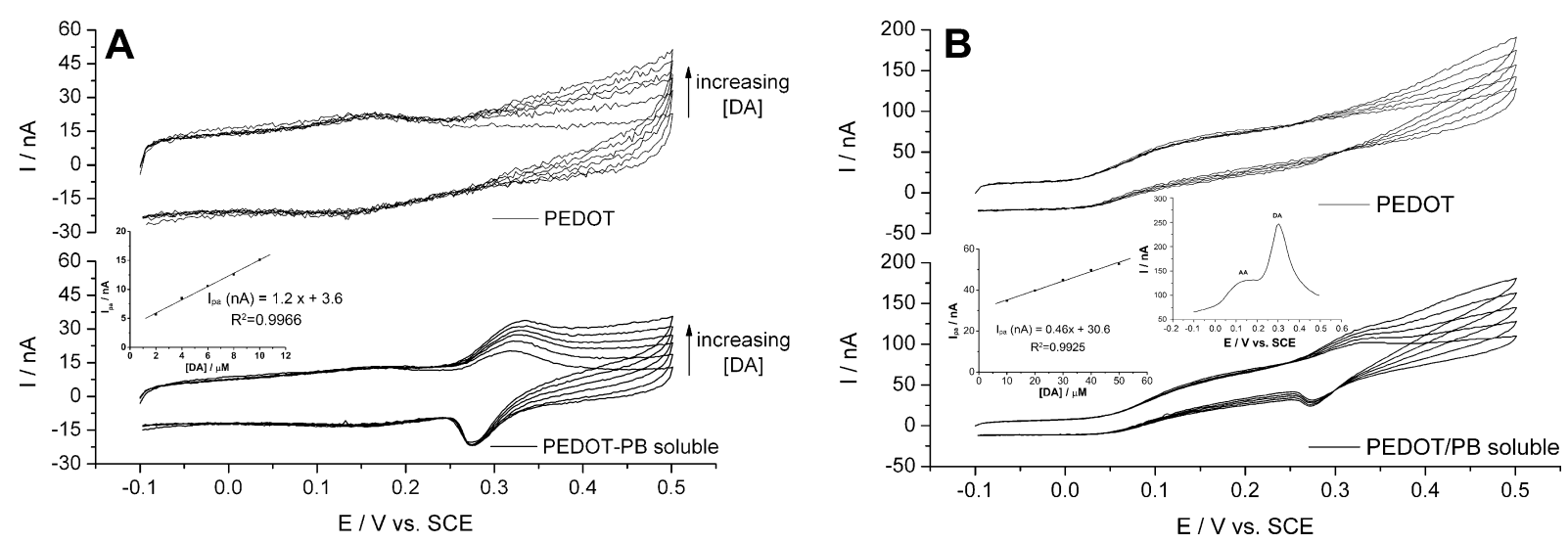

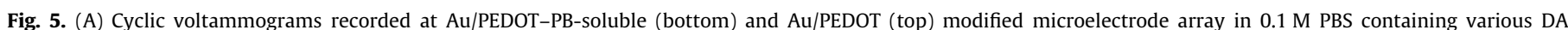

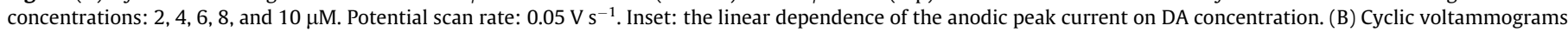

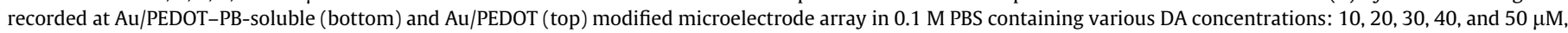

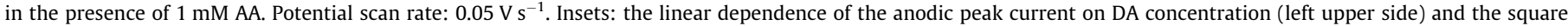

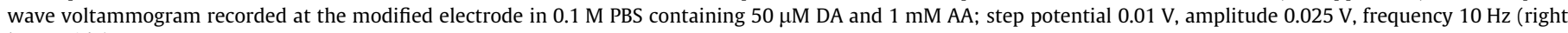
lower side).

behaviour of the organic and inorganic components of the composite material. The oxidation of DA at this modified electrode is reversible, while $A A$ oxidation is irreversible as it can be seen from Fig. 5A. The shift of peak potentials towards negative values attests the electrocatalytic activity of the composite material. The inorganic redox mediator, i.e. $\mathrm{PB}$, is able to mediate the oxidation of $\mathrm{DA}$ at the same potential values corresponding to the $\mathrm{PB} / \mathrm{ES}$ redox system. In the same time, the polarons from the PEDOT matrix are able to mediate the oxidation of AA and to assure the peak potential separation between the anodic waves of the analytes. This behaviour is based on the electrochemical properties of the composite materials, which combine the excellent stability of PEDOT in aqueous solution and its permselective membrane like behaviour with the good electrocatalytic activity of PB. The choice of these components in the fabrication of the composite material was based on the singular properties of each component as well as on the synergetic properties that could be expected.

A linear response of the sensor array modified with PEDOT-PBsoluble coating towards DA oxidation, in the presence of AA, was obtained over a concentration range from 10 to $50 \mu \mathrm{M}$ DA (see inset of Fig. 5B). The corresponding linear equation was: $i_{\mathrm{pa}}(\mathrm{nA})=$ $0.46 C_{\mathrm{DA}}(\mu \mathrm{M})+30.6$, with a correlation coefficient $R^{2}=0.9925$ (see inset of Fig. 5B), and a detection limit of $4.3 \mu \mathrm{M}$. The slopes of the calibration graphs, i.e. the $i_{\mathrm{pa}}$ vs. DA concentration plots in the absence and the presence of the interfering species, respectively, indicates that the presence of AA influences the DA oxidation. This is in agreement with data reported in the literature concerning the simultaneous voltammetric determination of DA, over a concentration range from 1 to $30 \mu \mathrm{M}$, in the presence of AA [29].

The electrocatalytic activity of PEDOT-PB-insoluble coating towards DA oxidation in the presence of AA was also investigated. In this case there is no peak shaped anodic wave for the oxidation of either analyte. Furthermore, the shape of the voltammograms is similar to those recorded at unmodified microelectrode array, and there is no improvement of the analytical performance. Hence the PEDOT-PB-insoluble modified electrode is not suitable for analytical applications.

\section{Conclusions}

Composite inorganic-organic coatings of various compositions were prepared, i.e. containing both soluble ad insoluble form of
PB. The deposition procedures reported allow the preparation in situ of both forms of $\mathrm{PB}$ as well as their electrochemical properties in electrolyte solutions. The PEDOT-PB-soluble composite material exhibited good electrochemical properties and electrocatalytic activity towards dopamine oxidation. The PEDOT-PB-insoluble coating showed no further improvement of the analytical performances with respect to the unmodified microelectrode array. The PEDOT organic coating as well as the PEDOT-PB-soluble composite coating showed good stability in aqueous buffered solution. The composite coating revealed to be porous enough to permit the free flux of potassium ions. The stability of the PEDOTPB-soluble composite material results from the electrostatic interaction between polarons in PEDOT and the negatively charge inorganic redox mediator. Low detection limit and linear responses over wide ranges of analyte concentration in the presence of a strong interferent have been obtained in the case of the PEDOTPB-soluble coating. The good electrochemical and electrocatalytic properties of this inorganic-organic composite material could be exploited in the construction of new microelectrochemical sensors as well as in similar analytical devices containing multiple sensing elements, as in electronic tongues.

\section{Acknowledgements}

S.L. acknowledges the financial support from the Romanian Ministry of Education and Research (MEdC). JdC acknowledges financial support from the Spanish Ministry of Science and Innovation (MICINN) through a Ramón y Cajal Fellowship.

\section{References}

[1] R.W. Murray (Ed.), Molecular Design of Electrode Surfaces, vol. 22, Wiley, New York, 1992.

[2] J. Roncali, J. Mater. Chem. 9 (1999) 1875-1893.

[3] M. Gerard, A. Chaubey, B.D. Malhotra, Biosens. Bioelectron. 17 (2002) 345-359.

[4] N.K. Guimard, N. Gomez, C.E. Schmidt, Prog. Polym. Sci. 32 (2007) 876-921.

[5] U. Lange, N.V. Roznyatovskaya, V.M. Mirsky, Anal. Chim. Acta 614 (2008) 1-26.

[6] A.A. Karyakin, Electroanalysis 13 (2001) 813-819.

[7] I.L. de Mattos, L. Gorton, T. Ruzgas, Biosens. Bioelectron. 18 (2003) 193-200.

[8] F. Ricci, G. Palleschi, Biosens. Bioelectron. 21 (2005) 389-407.

[9] S. Lupu, Rev. Roum. Chim. 50 (2005) 207-211.

[10] S. Lupu, Rev. Roum. Chim. 50 (2005) 213-217.

[11] K. Itaya, H. Akahashi, S. Toshima, J. Electrochem. Soc. 129 (1982) 1498-1500.

[12] K. Itaya, N. Shoji, I. Uchida, J. Am. Chem. Soc. 106 (1984) 3423-3429.

[13] L. He, C.-S. Toh, Anal. Chim. Acta 556 (2006) 1-15.

[14] P.J. Kulesza, K. Miecznikowski, M. Malik, M. Gałkowski, M. Chojak, K. Caban, A. Wieckowski, Electrochim. Acta 46 (2001) 4065-4073. 
[15] S.N. Sawant, N. Bagkar, H. Subramanian, J.V. Yakhim, Philos. Mag. 11 (2004) 2127-2138.

[16] A. Lisowska-Oleksiak, A.P. Nowak, V. Jasulaitiene, Electrochem. Comm. 8 (2006) 107-112.

[17] A. Lisowska-Oleksiak, A.P. Nowak, J. Power Sources 173 (2007) 829-836.

[18] M. Dietrich, J. Heinze, G. Heywang, F. Jonas, J. Electroanal. Chem. 369 (1994) 87-92.

[19] C. Kvarnstrom, H. Neugebauer, S. Blomquist, H.J. Ahonen, J. Kankare, A. Ivaska, Electrochim. Acta 44 (1999) 2739-2750.

[20] L. Adamczyk, P.J. Kulesza, K. Miecznikowski, B. Palys, M. Chojak, D. Krawczyk, J. Electrochem. Soc. 152 (2005) E98-E103.

[21] F. Li, S. Dong, Electrochim. Acta 32 (1987) 1511-1513.
[22] A. Ernst, O. Makowski, B. Kowalewska, K. Miecznikowski, P.J. Kulesza, Bioelectrochemistry 71 (2007) 23-28.

[23] R. Koncki, Crit. Rev. Anal. Chem. 32 (2002) 79-96.

[24] O. Ordeig, C.E. Banks, F.J. del Campo, F.X. Munoz, J. Davis, R.G. Compton, Electroanalysis 18 (2006) 247-252.

[25] V.S. Vasantha, S.-M. Chen, Electrochim. Acta 51 (2005) 347-355.

[26] S. Lupu, P.C. Balaure, C. Lete, M. Marin, N. Totir, Rev. Roum. Chim. 53 (2008) 931-939.

[27] K. Itaya, I. Uchida, V.D. Neff, Accounts Chem. Res. 19 (1986) 162-168

[28] S. Lupu, N. Totir, C. Mihailciuc, C. Lete, Rev. Roum. Chim. 48 (2003) 39-43.

[29] S. Senthil Kumar, J. Mathiyarasu, K.L.N. Phani, V. Yegnaraman, J. Solid State Electrochem. 10 (2006) 905-913. 